\title{
Space-time Structure of Initial Parton Production in Ultrarelativistic Heavy Ion Collisions
}

\author{
K. J. Eskola ${ }^{1,2}$ and Xin-Nian Wang ${ }^{1}$ \\ ${ }^{1}$ Nuclear Science Division, Mailstop 70A-3307, Lawrence Berkeley Laboratory \\ University of California, Berkeley, California 94720. \\ ${ }^{2}$ Laboratory of High Energy Physics, P.O. Box 9 \\ SF-00014 University of Helsinki, Finland.
}

(Received )

\begin{abstract}
The space and time evolution of initial parton production in ultrarelativistic heavy ion collisions is investigated within the framework of perturbative QCD which includes both initial and final state radiations. Uncertainty principle is used to relate the life time of a radiating parton to its virtuality and momentum. The interaction time of each hard or semihard parton scattering is also taken into account. For central $A u+A u$ collisions at $\sqrt{s}=200 \mathrm{GeV}$, most of the partons are found to be produced within $0.5 \mathrm{fm} / \mathrm{c}$ after the total overlap of the two colliding nuclei. The local momentum distribution is approximately isotropical at that time. The implication on how to treat correctly the the secondary scattering in an ultimate parton cascading model is also discussed.
\end{abstract}

25.75.+r, 12.38.Mh, 13.87.Ce, 24.85.+p

Typeset Using REVTEX 
In the search for a quark gluon plasma (QGP), a thermally and chemically equilibrated system of quarks and gluons freed from the color confinement, heavy nuclei are accelerated to collide with each other at ultrarelativistic energies. Studies in the last few years [1]- [5] suggest that heavy ion collisions are dominated by minijet production via semihard parton scatterings with $p_{T} \sim$ few $\mathrm{GeV} / \mathrm{c}$ at energies of the Relativistic Heavy Ion Collider (RHIC) of Brookhaven National Laboratory (BNL) and the proposed Large Hadron Collider (LHC) of CERN. These minijets may contribute to most of the transverse energy produced in the heavy ion collisions. If there is enough time and if the density is sufficiently high for the partons to rescatter, the initially produced minijets will eventually lead to a thermalized QGP. However, in order to estimate the initial energy density of the produced partons, one must know their formation time. The total formation time must include the interaction time of the semihard processes and the formation time of initial and final state radiation.

Another motivation of our study in this paper is to understand the space-time evolution of the initial parton production and the consequences on secondary scatterings. One has to consider these scatterings in order to simulate the thermalization process by parton cascading. Although there are already Monte Carlo simulations of parton cascading in heavy ion collisions [4, 5], the possibility of double counting is still a potential problem. One type of double counting may occur if one neglects the interaction time of the scatterings. During the interaction time, which depends on whether the scatterings is hard, semihard or soft, the participating and therefore the produced partons cannot scatter again immediately with the beam partons. Another double counting has to do with the initially radiated partons before the hard or semihard scatterings. In calculating jet cross sections and the number of hard scatterings, parton structure functions of a nucleus $f_{a / A}\left(x, Q^{2}\right)$ evaluated at the scale $Q^{2}=p_{T}^{2}$ are used. These parton distribution functions then have already included the effect of QCD evolution, producing more partons at small $x$ and larger $Q^{2}$. Therefore, in Monte Carlo simulations in which initial radiations are treated as backward evolution, the radiated partons should not participate in any interactions before the end of the corresponding hard scattering. This is also in coincidence with the factorization theorem [6] of perturbative QCD (pQCD).

In this paper, we will use HIJING Monte Carlo model [3], which was developed for simulating parton and particle production in heavy ion collisions, to study the space-time evolution of the initial parton production. In HIJING, each hard parton 
scattering in hadronic reactions is simulated by using some subroutines of PYTHIA [7] in which one can trace back the whole history of initial and final state radiation associated with that hard scattering. The life time of an intermediate off-shell parton is estimated via the uncertainty principle $t_{q} \sim E / q^{2}$ for given energy $E$ and virtuality $q$. We then obtain the space and time vertices of all the produced partons. Given a space cell, we then calculate the time evolution of the momentum distribution of the produced partons by assuming classical trajectories without rescatterings. We will verify that after some time most of the partons will leave the space cell except those whose rapidities are close to the spatial rapidity of the cell, thus achieving local isotropy in momentum distribution which happens to look like thermal. This will provide a good starting point for treating equilibration via a set of rate equations as in Refs. 8,9] for the number and energy densities. However, what is not studied in this paper, though included in HIJING, is the particle production from the soft component.

This paper is organized as follows: In Sec. I, we briefly review the HIJING model and the related aspects of parton shower in PYTHIA. More detailed descriptions can be found in Refs. [3, 7]. In Sec. II, we discuss how we implement the Lorentz contracted spatial parton distribution of a nucleus into the calculation and how we estimate the life time of a virtual parton and the interaction time of semihard processes. The results are presented and discussed in Sec. III, where consequences for secondary parton scattering and thermalization processes are also discussed. We give our conclusions in Sec. IV.

\section{HIJING MODEL}

To make a consistent study of the initial parton production, we briefly review the HIJING model and the structure of initial and final state radiation in PYTHIA which HIJING model utilizes exclusively for hard parton scatterings and the associated radiations. Readers can find detailed descriptions in Refs. [3],7].

\section{A. Minijet Production}

In perturbative QCD, given the parton structure functions $f_{a / N}\left(x, Q^{2}\right)$ and the perturbative parton-parton cross section $d \sigma_{a b}$, the differential cross section for jet production in nucleon-nucleon collisions can be calculated via [10] 


$$
\frac{d \sigma_{\mathrm{jet}}}{d p_{T}^{2} d y_{1} d y_{2}}=K \sum_{\substack{a b \\ c d}} x_{1} f_{a / N}\left(x_{1}, p_{T}^{2}\right) x_{2} f_{b / N}\left(x_{2}, p_{T}^{2}\right){\frac{d \sigma^{a b \rightarrow c d}}{d \hat{t}}}^{a b}
$$

where $x_{1,2}$ are the fractional momenta of the colliding partons, $y_{1,2}$ are the rapidities of the jets and $p_{T}$ is their transverse momentum. The factor $K \approx 2$ accounts roughly for the corrections beyond the leading order. We want to emphasize here that the jet cross sections are calculated with the parton structure functions evaluated at the scale of the hard scattering $Q^{2}=p_{T}^{2}$. These structure functions $f_{a / N}\left(x, Q^{2}\right)$ are obtained by evolving them from a lower scale $Q_{0}^{2}$ via Altarelli-Parisi equations [11] thus increasing the parton density at lower values of $x$.

Given the integrated inclusive jet cross section $\sigma_{\text {jet }}$, we can use the unitarized eikonal model [12]- [17] to calculate the total nucleon-nucleon cross section and the cross sections for multiple independent jet production 18]

$$
\begin{aligned}
\sigma_{0} & =\int d^{2} b\left[1-e^{-\sigma_{\mathrm{soft}} T_{N}(b)}\right] e^{-\sigma_{\mathrm{jet}} T_{N}(b)}, \\
\sigma_{j} & =\int d^{2} b \frac{\left[\sigma_{\mathrm{jet}} T_{N}(b)\right]^{j}}{j !} e^{-\sigma_{\mathrm{jet}} T_{N}(b)},
\end{aligned}
$$

where $T_{N}(b)$ is the partonic overlap function between two colliding nucleons at impact parameter $b$.

In this model two phenomenological parameters have to be introduced [18]: An infrared cutoff $p_{0}=2 \mathrm{GeV} / \mathrm{c}$ is used to calculate the total inclusive "hard" parton interaction cross section $\sigma_{\text {jet }}\left(p_{0}, \sqrt{s}\right)$. Another parameter, $\sigma_{\text {soft }}=57 \mathrm{mb}$, is used to characterize the corresponding "soft" parton interactions. Most of the differences among several existing models [3 [5] result from different values of these two parameters used. As demonstrated in Ref. [18], these two parameters, though constrained by the total $p p$ and $p \bar{p}$ cross sections, are still model dependent. Since most of the minijets are nonresovable as distinct hadronic clusters in the calorimeter of an experimental detector, their existence can only be justified by their contribution to particle production in low and intermediate $p_{T}$ region. Therefore, the value of $p_{0}$ and $\sigma_{\text {soft }}$ will depend on what is included in the particle production from the so-called "soft" interactions. Recently, two-particle correlation functions in azimuthal angle and their energy and $p_{T}$ dependence are proposed [19] to give further constraints on these two parameters.

Extrapolated to heavy ion collisions which are decomposed into binary nucleonnucleon scatterings, Eqs.(2).3) are used to determine the number of minijet production in each binary collision. Each hard scattering is then simulated via subroutines of 
PYTHIA and initial and final state radiations are generated. At ultrarelativistic energies, the colliding nuclei are highly contracted longitudinally in the center-of-mass frame but are surrounded by soft partons with the same longitudinal size as a nucleon. The hard or semihard scatterings all happen at around the same time when the two nuclei pass through each other. Therefore, binary collision is a good approximation for hard scatterings. In each binary collision the same parton structure can be used. In other words, each individual nucleon really does not have time to readjust its parton distribution before the next binary collision. Of course, in the actual simulation, energy and momentum conservation is strictly imposed. To take into account the nuclear modification of the parton structure functions, we parameterize [3] the nuclear shadowing effect as measured experimentally in deeply inelastic lepton-nucleus scatterings and assume gluons and quarks are shadowed by the same amount in small $x$ region. The inclusion of nuclear shadowing will effectively reduce the number of minijet production by about \%50 at RHIC energy. However, considering the QCD evolution of the shadowing [20] may reduce the effective shadowing.

\section{B. Initial and Final State Radiation}

For each hard scatterings, one then has to take into account the corrections due to initial and final state radiations. In an axial gauge and to the leading pole approximation, the interference terms of the radiation drop out. The amplitude for successive radiations has then a simple ladder structure and the probability for multiple emission becomes the product of each emission [21. The virtualities of the radiating partons are ordered along the tree, decreasing until a final value $\mu_{0}^{2}$ below which pQCD is no longer valid any more. This provides a framework for a Monte Carlo simulation of parton shower and its space-time interpretation [22,23.

At a given vertex of the branching tree, the probability for the off-shell parton $a$ of virtuality $q^{2}<q_{\max }^{2}$ to branch into partons $b$ and $c$ with fractions $z$ and $1-z$ of the light-cone momentum is given by 22, 23]

$$
d \mathcal{P}_{a \rightarrow b c}\left(q^{2}, z\right)=\frac{d q^{2}}{q^{2}} d z P_{a \rightarrow b c}(z) \frac{\alpha_{s}\left[z(1-z) q^{2}\right]}{2 \pi} \frac{\mathcal{S}_{a}\left(q_{\text {max }}^{2}\right)}{\mathcal{S}_{a}\left(q^{2}\right)}
$$

where $P_{a \rightarrow b c}(z)$ is the Altarelli-Parisi splitting function [11] for $a \rightarrow b c$ process. By requiring the relative transverse momentum $q_{T}$ of $b$ and $c$ to be real,

$$
q_{T}^{2}=z(1-z)\left(q^{2}-\frac{q_{b}^{2}}{z}-\frac{q_{c}^{2}}{1-z}\right) \geq 0
$$


and a minimum virtuality $q_{b}^{2}, q_{c}^{2} \geq \mu_{0}^{2}$, the kinematically allowed region of phase space is then,

$$
\begin{aligned}
& 4 \mu_{0}^{2}<q^{2}<q_{\max }^{2} \\
& \epsilon(q)<z<1-\epsilon(q), \quad \epsilon(q)=\frac{1}{2}\left(1-\sqrt{1-4 \mu_{0}^{2} / q^{2}}\right) .
\end{aligned}
$$

The Sudakov form factor $\mathcal{S}_{a}\left(q^{2}\right)$ is defined as

$$
\mathcal{S}_{a}\left(q^{2}\right)=\exp \left\{-\int_{4 \mu_{0}^{2}}^{q^{2}} \frac{d k^{2}}{k^{2}} \int_{\epsilon(k)}^{1-\epsilon(k)} d z \sum_{b, c} P_{a \rightarrow b c}(z) \frac{\alpha_{s}\left[z(1-z) k^{2}\right]}{2 \pi}\right\},
$$

so that $\mathcal{S}_{a}\left(q_{\text {max }}^{2}\right) / \mathcal{S}_{a}\left(q^{2}\right)$ is the probability for parton a not to have any branching between $q_{\max }^{2}$ and $q^{2}$.

In principle, one can perform the initial state radiation in a similar way. The partons inside a nucleon can initiate a space-like branching increasing their virtuality from some initial value $Q_{0}^{2}$. A hard scattering can be considered as a probe which can only resolve partons with virtuality up to the scale of the hard scattering. Otherwise without the scattering, the off-shell partons are only virtual fluctuations inside the hadron and they will reassemble back to the initial partons. In PYTHIA, which uses backward evolution, a hard scattering is selected first with the known QCDevolved structure function at that scale, and then the initial branching processes are reconstructed down to the initial scale $Q_{0}^{2}$. The evolution equations are essentially the same as in final state radiation except that one has to convolute with the parton structure functions. Readers can find details in Ref. [7].

\section{FORMATION AND INTERACTION TIME}

When a parton is off-shell, it can be considered as a virtual fluctuation and it can only live for a finite time, $\Delta t$, determined by its virtuality $q^{2}$ via the uncertainty principle,

$$
\Delta t \approx q_{0} / q^{2}
$$

where $q_{0}$ is the energy of the parton. After $\Delta t$, the off-shell parton will then branch or "decay" into other partons which can further initiate branchings until a minimum virtuality $\mu_{0}$ is reached. At $q^{2} \leq \mu_{0}^{2}$, pQCD is not considered to be valid anymore and the process of nonperturbative hadronization takes over. Following this tree of branching (which also includes initial space-like radiation) and assuming a straight 
line trajectory for partons, we can then calculate the space and time evolution of the initial parton production. Since we don't consider secondary parton-parton interactions, the produced final partons which are on shell are considered free particles. Fig. 1 is a demonstration of a hard scattering and the associated branching trees. The number on each line represents the life time (in $\mathrm{fm} / \mathrm{c}$ ) of that virtual parton and the numbers in parentheses are the corresponding virtualities (in $\mathrm{GeV}$ ).

The life time of a virtual parton in Eq. 8 is only a rough estimate according to the uncertainty principle. One could also estimate it via pQCD in lowest order [4] which gives an extra factor of $1 / \alpha_{s}$. But higher order corrections could easily cancel this factor, so that $q_{0} / q^{2}$ should be a good estimate of $\Delta t$ in magnitude.

We also use Eq. 8 to estimate the interaction time for each hard scattering with $q$ being the sum of the initial or final four-momentum of the colliding partons. If the fractional momenta of the partons are $x_{1}$ and $x_{2}$, then the interaction time can be estimated as

$$
\Delta t_{i} \approx \frac{x_{1}+x_{2}}{2 x_{1} x_{2} \sqrt{s}}
$$

In this case, the asymmetric scatterings $\left(x_{1} \gg x_{2}\right.$ or $\left.x_{1} \ll x_{2}\right)$ have longer interaction time than the symmetric ones $\left(x_{1} \sim x_{2}\right)$ for fixed parton-parton center-of-mass energy $x_{1} x_{2} \sqrt{s}$. We also assume that the interaction time is the same for all channels.

In the rest frame of each nucleus, three-parameter Wood-Saxon nuclear densities are used to construct the nucleon distribution inside the nucleus. The system is then boosted to the center-of-mass frame of the two colliding nuclei. Due to the fact that gluons, sea quarks and antiquarks are only quantum fluctuations before they really suffer scatterings, their longitudinal distribution around the center of the nucleon is still governed by the uncertainty principle in any boosted frame [24]. We refer to this distribution as the "contracted distribution", in which a parton with $x_{i}$ fractional momentum has a finite spatial spread,

$$
\Delta z_{i} \approx 2 / x_{i} \sqrt{s}
$$

Transversely, partons are distributed around their parent nucleons according to the Fourier transform of a dipole form factor 18. If we define $t=0$ as the moment when the two nuclei have complete overlap, then the interaction point of two partons in a $t-z$ plane can be anywhere within the shaded area in Fig. 2. The solid lines are the trajectories of the two parent nucleons which spread around the nuclei according to a longitudinally contracted Wood-Saxon distribution. 
Because of the spread of parton interaction points in space and time, it is ambiguous to define a common proper time in the $t-z$ plane for parton production in heavy ion collisions. Therefore, as we will demonstrate in the next section, a proper time,

$$
\tau=\sqrt{t^{2}-z^{2}}
$$

defined with respect to the nucleus overlap point, is only approximately relevant.

\section{NUMERICAL RESULTS}

In this section, we present the numerical results of our study. All our results are for central $A u+A u$ collisions at RHIC energy. In our simulation, Duke-Owens parton distributions [25] for nucleons are used. The initial virtuality for the initial state evolution is set to be $Q_{0}=2 \mathrm{GeV} / \mathrm{c}$, and the minimum virtuality for the final state radiation is $\mu_{0}=0.5 \mathrm{GeV} / \mathrm{c}$. The maximum virtuality for the associated radiations in a hard scattering with transverse momentum transfer $p_{T}$ is chosen to be $q_{\max }=2 p_{T}$. In PYTHIA, angular ordering is also enforced to take into account the soft gluon interference 23] in the final state radiation. In HIJING model, soft interactions as string excitations are also included. These soft interactions must also consume energy and affect minijet production slightly. We want to emphasize that in our calculation here, we do not include the soft interactions, so the number of minijet production here should be slightly more than the full calculation when soft interactions are included.

\section{A. Space-time Evolution}

To estimate the parton production time, we plot in Fig. 3 the total number of produced partons, on-shell as well as off-shell, as a function of time at the highest RHIC energy, $\sqrt{s}=200 \mathrm{GeV} / \mathrm{n}$. We see that long before the two nuclei overlap and hard scatterings take place, partons have already been produced via initial state bremsstrahlung. Some of the initially radiated partons will also initiate time-like branching trees(see Fig.11). Note that, if the coherence is not broken by the hard scattering, partons which would have been emitted from the initial state radiation will not emerge as produced partons. Here we have also included the initiators of the space-like branching as produced partons. Therefore, if a parton does not have initial state radiation, it will only become a produced parton after the hard scattering, 
whereas, a parton is defined to be produced before the hard scattering if it has initial state radiation. From Fig. 3, we can see that about $2 / 3$ of the total number of partons are produced between $t=-0.5$ and $0.5 \mathrm{fm} / \mathrm{c}$ while about 200 semihard scatterings happen between $t=-0.1$ and $0.1 \mathrm{fm} / \mathrm{c}$ as indicated by dashed lines. We find also that about $2 / 3$ of the total number of partons are produced in initial and final state radiations. The fraction of partons from branching should increase with the colliding energy and with smaller choices for $\mu_{0}$.

To see how hard or semihard scatterings and initial and final state bremsstrahlung contribute to parton production, we show in Fig. 14 the rapidity distribution of produced partons at different time. Before $t=0$, most of the partons come from initial state radiation. Since the radiations are almost collinear, these partons move along the beam direction and therefore have large rapidities. The semihard scatterings then produce partons uniformly over a rapidity plateau and fill up the middle rapidity region. Final state radiations, which happen after the semihard scatterings, will also produce partons uniformly in the central rapidity region. We therefore see from Fig. 4 that the dip of $d N / d y$ in middle rapidity is actually caused by the parton production from initial state bremsstrahlung at large rapidity. These partons with large longitudinal momenta will move away from the interaction region after the semihard scatterings. They do not rescatter with the beam partons in the leading twist approximation. We will come back to this point again when we discuss the consequences of our study on how to treat parton rescattering. In Fig. 5, we also show the time evolution of the $p_{T}$ distribution of the produced partons. Since the partons, which initially have a Gaussian $p_{T}$ distribution, have gone through initial state radiation, they have already a large $p_{T}$ tail at $t=-0.6 \mathrm{fm} / \mathrm{c}$ before the hard scattering. Hard scatterings will transfer large transverse momentum to the final partons and the timelike branchings produce a lot of partons with small $p_{T}$. The final $p_{T}$ spectrum then looks more or less like an exponential one, even though secondary scatterings have not yet been taken into account.

In Fig. 6, we plot $d N / d z$ as a function of $z$ at different time to illustrate how the parton production evolves in space and time. At $t=-0.7 \mathrm{fm} / \mathrm{c}$, as the two nuclei approach toward each other before they actually overlap, initial state radiations have already begun. These partons have large rapidities and are Lorentz contracted with an average spread in $z$,

$$
\Delta z \approx 1 / p_{0}+2 R_{A} \frac{2 m_{N}}{\sqrt{s}} \approx 0.25 \mathrm{fm}, \quad \sqrt{s}=200 \mathrm{GeV},
$$

where $p_{0}=2 \mathrm{GeV} / \mathrm{c}$ is the $p_{T}$ cutoff for semihard scattering, $R_{A}$ is the nuclear radius 
of $A u$ and $m_{N}$ is the nucleon mass. After the hard scatterings and during the interaction time, partons are produced uniformly in the central rapidity region. Afterwards, partons follow straight line by free-streaming and are distributed evenly in $z$ between two receding pancakes of beam partons (partons from initial state radiations). One can clearly see that there is approximately boost invariance in the central region in this free-streaming picture.

Another illustrative way to study the evolution of local parton density is to make a contour plot of parton density $\rho$ in $z$ and $t$ as shown in Fig. 7. Here, $\rho$ is defined as

$$
\rho=\frac{1}{\pi R_{A}^{2}} \frac{d N}{d z}
$$

and a sharp sphere distribution is assumed for nuclear density. One can clearly see that partons inside the two approaching nuclei have a spatial spread of $\Delta z=0.25$ $\mathrm{fm}$ in $z$. This spread continues for partons from the initial state radiation as they escape from the interaction region along the beam direction with large rapidities. The interaction region where semihard scatterings happen lasts for about $0.5 \mathrm{fm} / \mathrm{c}$, from $t=-0.25$ to $0.25 \mathrm{fm} / \mathrm{c}$. Because of the contracted distribution, some of the partons lie outside the light-cone which is defined with respect to the overlapping point of the two nuclei. The definition of a proper time $\tau$ (Eq. 11) for the evolution of the whole system is therefore only valid within an accuracy of $\Delta z$.

If one assumes boost invariance [26], the parton density can be estimated as

$$
\rho=\frac{1}{\pi R_{A}^{2} \tau} \frac{d N}{d y}
$$

where $\tau$ is the proper time and $\rho$ should be a function of $\tau$ only. By comparing the contour of constant density with the hyperbola of constant $\tau$ (dot-dashed line) in Fig. 7, we see that this is true only approximately. We also see that the density, as indicated by the numbers, decreases like $1 / \tau$ due to free-streaming.

\section{B. Local Isotropy in Momentum Space}

Since there are numerous partons produced within a rather short time as we have demonstrated, the initial parton density is very high at $t \geq 0.25 \mathrm{fm} / \mathrm{c}$, immediately after the interaction region (see Fig. 7). Within such a dense system, secondary parton scatterings and production are inevitable. The equilibration time for the system can be estimated by solving a set of rate equations as recently has been done in Ref. [8]. In this approach, one must make sure that there is approximately local isotropy in 
momentum space. This could be achieved through secondary parton scatterings as has been investigated in Refs. [4, 27]. One can also use free-streaming to estimate the upper bound of the thermalization time $t_{\text {iso }}$ [8, 28], by studying the momentum distribution of partons in a cell of the size of the mean-free-path $\lambda_{f}$. We take $\lambda_{f} \sim 1$ fm in our study here.

Let's concentrate on the central slice at $z=0$ with $|z|<0.5 \mathrm{fm}$. At the very early stage during the interaction region (for example at $t=0$ in Fig. 母), produced partons with different rapidities are confined to a highly compressed slab with $\Delta z \approx 0.5 \mathrm{fm}$. As the system expands, partons with large rapidities will escape from the central slice while partons with small rapidities remain. As shown in Fig. 8, the rapidity distribution of the produced partons in this central slice evolves from a plateau-like distribution with width $\Delta y / 2 \approx 2.5$ at early times to a Gaussian shape at $t=0.7$ $\mathrm{fm} / \mathrm{c}$. If the free-streaming continues, the rapidity distribution in this central slice will eventually become a $\delta$-function and only partons with zero rapidity remain. The evolution in other cells is similar in their local frames which have spatial rapidity

$$
\eta=\frac{1}{2} \ln \frac{t+z}{t-z}
$$

In Fig. 9, we show the evolution of momentum distributions in $p_{x}$ (solid lines) and $p_{z}$ (dashed lines) at different times (indicated by the number on each line). The $p_{x}$ distribution should evolve like $p_{T}$ distribution as is shown in Fig. 5. The slope of $p_{z}$ distribution, however, decreases because partons with large longitudinal momenta gradually escape from the central region. At $t=0.7 \mathrm{fm} / \mathrm{c}$, the slopes of $p_{x}$ and $p_{z}$ distributions become the same. If one wants to use the parton production we have calculated so far as an initial condition to study thermal and chemical equilibration, this should be the starting point where local isotropy is approximately achieved. Therefore, the time for achieving isotropy in momentum space via free-streaming is

$$
t_{\mathrm{iso}} \approx 0.7 \mathrm{fm} / \mathrm{c} \text {. }
$$

This is the same as estimated in Ref. [8]. We emphasize that this is only an estimate of the upper bound. However, without secondary scatterings, thermalization can never be achieved and maintained.

\section{Chemical Composition}

Unlike an ideal gas of quarks and gluons in which chemical equilibrium is maintained, the initial production of quarks and gluons is determined by the parton structure functions, the hard scattering cross sections and the radiation processes in pQCD. 
Due to the difference in the numbers of degrees of freedom in color space, cross sections involving gluons are always larger than those of quarks. For small angle scatterings, one can verify that,

$$
\frac{d \sigma_{i}}{d t} \cong C_{i} \frac{2 \pi \alpha_{s}^{2}}{t^{2}}
$$

where $t$ is one of the Mandelstam variables and,

$$
C_{i}=\frac{4}{9}, 1, \frac{9}{4}
$$

for $i=q q, g q$ and $g g$ scatterings. Similarly, both initial and final state radiations produce more gluons than quarks and anti-quarks. Therefore in pQCD, the ratio between produced quarks and gluons is much smaller than the ratio of an ideal gas, which is 9/4 for three quark flavors. Shown in Fig. 10 are the fractions of produced quarks and anti-quarks as functions of time $t$ in $p p$ and $A A$ collisions at $\sqrt{s}=200$ $\mathrm{GeV} / \mathrm{n}$. For $A u+A u$ collisions, hard scatterings with $p_{T}>2 \mathrm{GeV} / \mathrm{c}$ produce about $13 \%$ quarks and anti-quarks. If initial and final state radiations are not included the ratio jumps to $18 \%$, because radiations produce more gluons than quarks and antiquarks. In $p p$ collisions, about $28 \%$ of the partons produced via hard scatterings without radiations are quarks and anti-quarks. The difference between $A u+A u$ and $p p$ collisions is due to the different $A$ dependence of the valence and sea quark production. Since the partons are produced via binary collisions, the number of produced gluons and sea quarks and antiquarks scales like $A^{4 / 3}$. On the other hand, baryon number conservation requires valence quark production to scale like $A$. Given the fraction of total quark $q_{p p}=0.28$ and valence quark $v_{p p}=0.14$ production in $p p$ collisions, one can find for $A u+A u$ collisions,

$$
q_{A A}=\frac{q_{p p}-\left(1-A^{-1 / 3}\right) v_{p p}}{1-\left(1-A^{-1 / 3}\right) v_{p p}}
$$

which gives $q_{A A}=0.18$ for $A=197$ as we obtained from Fig. 10 of the numerical calculation. To demonstrate the effective valence quark production, we plot in Fig. 11 the rapidity dependence of the fractional quark number. In $p p$ collisions, the valence quark production peaks at large rapidities. In nucleus-nucleus collisions, the valence quark production has a different scaling in $A$ than gluons and sea quarks. This is why the fraction of quark and antiquark production is suppressed more at large rapidity than in the central rapidity region. The relative quark and antiquark production in the central rapidity region is further suppressed by final state radiation.

The small fractional quark production within pQCD has important consequences for chemical equilibration of the partonic system. Because of the small initial relative 
quark density and small quark production cross section as compared to gluon production, it takes a very long time, if ever, for the system to achieve chemical equilibrium. If this time is longer than the phase transition time, a fully equilibrated QGP may never be formed 8 .

\section{Consequences on Parton Rescatterings}

So far we have not considered final state interactions among the produced partons. Though an exact quantum field treatment of parton cascading is not possible with present technology, various semi-classical approximations has been undertaken [四, 5]. The most important and difficult effort in these semiclassical approximations is to emulate the quantum effects, like interference, coherence, and especially, nonperturbative phenomena in QCD. Since we have studied the initial parton production, a discussion of the consequences on how to treat final state parton rescatterings seems to be in place now.

We have explicitly taken into account the interaction time for the semi-hard scatterings, which is roughly $t_{i} \sim 1 / p_{T}$. Inside a highly Lorentz contracted nucleus, the spatial spread for partons which could participate in a hard scattering with transverse momentum transfer $p_{T}$ is also about $\Delta z \sim 1 / p_{T}$. This would leave the produced partons no time to have another hard scattering of $p_{T}$ with the incoming beam partons. For finitely contracted nuclei at relatively low energies, this kind of double high $p_{T}$ scattering is still possible. However, these kind of higher twist processes should be suppressed by a factor of $1 / p_{T}^{2}$. It is also possible for a parton to go through a hard and a soft scattering subsequently since the soft partons always have a spatial spread of $1 \mathrm{fm}$. This kind of hard-soft multiple interactions constitute the leading contribution to higher twist corrections to hard processes in nuclear collisions [29]. However, double semihard scatterings at high energy with $p_{T} \sim p_{0}$ will be suppressed due to finite interaction time.

The large $p_{T}$ enhancement of both Drell-Yan dilepton production and single hadrons in $p A$ collisions at $\sqrt{s} \leq 50 \mathrm{GeV}$ is considered as a result of multiple parton interactions [30]. At these energies, the contracted length of a heavy nucleus is still relatively larger than the interaction and formation time. The factorized form of parton model is then modified due to the finite beam energy. However, a collection of experimental data [31,32] even in this intermediate energy range has already shown the effects of the finite interaction time. As the energy increases, the interaction time becomes more important as compared to the size of an increasingly contracted 
nucleus. The partons then have less time for secondary scatterings. This then leads to the observed decrease of the large $p_{T}$ enhacement in $p A$ interactions as the beam energy increases. At ultrarelativistic energies, one should therefore expect the suppression of double semihard scatterings.

In this study, we used a QCD-based probabilistic model to calculate the number of multiple independent semi-hard parton scatterings. When calculating the inclusive jet cross section in Eq. 1, we used the QCD-evolved parton structure function $f_{a / A}\left(x, Q^{2}\right)$ at the scale of the hard scattering $Q^{2}$. Therefore by sampling the partons from a joint distribution function of two colliding nuclei at $Q^{2}$,

$$
f_{a / A}\left(x_{1}, Q^{2}\right) f_{b / B}\left(x_{2}, Q^{2}\right)
$$

we have already included the probability for partons from initial state radiation to participate in the semihard scatterings. As illustrated in Fig. 12, the two partons which are chosen to have hard scatterings may originate from the same initial branching tree. Since not every parton has to have hard scattering in our probabilistic model, other partons produced from the initial state radiation tree have been chosen not to suffer hard scatterings. These partons should not be allowed to have further scatterings with the beam partons, even though they are produced long before the hard scattering and have to "go through" the interaction region during the space-time evolution (see Fig. (7). Another way to understand this is to consider all partons in the initial state radiation tree as fluctuations. During the hard scatterings, the beam partons act as probes which can resolve the fluctuations up to scale $Q^{2}$. These partons on the branching tree leading to the hard scatterings will become incoherent, while those not leading to any hard scattering will eventually reassemble back to the initiating partons. In our probabilistic model, the beam partons will be chosen to interact with only some of these resolved partons, while others will simply move along the beam direction even though they become incoherent to the nucleons.

In our simulation, partons in different hard scatterings have their own independent initial state radiation trees, decreasing the virtuality from $Q$ backward to an initial scale $Q_{0}$ below which everything is considered nonperturbative. This corresponds to the situation of $Q_{2}=Q_{0}$ in Fig. 12, where both parton fission and fusion are included in the nonperturbative regime. However, the two branching trees could also be correlated as in Fig. 12 if $Q_{1}=Q_{0}$. In this case, parton fusion and fission take place in the perturbative regime and the parton structure functions will be modified [33. One, therefore, should use multiple parton correlation function $f\left(x_{1}, \ldots, x_{i}, Q^{2}\right)$. In this paper, we simply used a parametrization [3] to take into account the correlation 
effect. If this is not enough, we might have overestimated the parton production from the initial state radiation.

\section{CONCLUSIONS}

We have studied the space-time evolution of initial parton production in ultrarelativistic heavy ion collisions. At RHIC energy, we found that the production time is about $0.5 \mathrm{fm} / \mathrm{c}$ after the two colliding nuclei have complete overlap. At $t=0.7$ $\mathrm{fm} / \mathrm{c}$, the produced partons inside a cell $|z| \leq 0.5 \mathrm{fm}$ have momentarily achieved local isotropy in momentum space by free-streaming, consistent with the estimate in Ref. [8]. This could provide a starting point if one wants to investigate the equilibration of the produced partons via rate equations. The initially produced partons are however far away from chemical balance due to small quark production cross sections.

The formation time of the produced partons has serious consequences on the subsequent secondary scatterings. The number of double semihard scatterings should be suppressed if one takes into account the formation time. Due to factorization, partons from the associated initial state radiation will also not scatter again with the incoming beam partons.

In hadronic collisions, minijets become important only at above $\sqrt{s}=50 \mathrm{GeV} / \mathrm{c}$. Soft interactions, however, still have important contributions to the total cross section and particle production even at the highest collider energy presently available. What we have calculated in this paper is only the parton production through the semihard processes. We have not included the nonperturbative soft processes. In HIJING model, the soft interactions have been modeled as string formation, which carries energy by means of a color field. Naively, one would think these soft interactions are still present in the final state cascading. However, as has been shown in Refs. [34, 9], the high partonic density produced by the semihard scatterings will screen the color field and make the soft component less important. What are left over may only be those semihard rescatterings among the produced partons, with the infrared cutoff replaced by the screening mass. Then we would use pQCD to simulate the parton cascading consistently. We hope the study in this paper will pave our way ultimately to such a cascading model. 
Discussions with M. Gyulassy are gratefully acknowledged. KJE thanks Magnus Ehrnrooth foundation and Suomen Kulttuurirahasto for partial financial support. This work was supported by the Director, Office of Energy Research, Division of Nuclear Physics of the Office of High Energy and Nuclear Physics of the U.S. Department of Energy under Contract No. DE-AC03-76SF00098. 
[1] K. Kajantie, P. V. Landshoff and J. Lindfors, Phys. Rev. Lett. 59, 2517 (1987);

K. J. Eskola, K. Kajantie and J. Lindfors, Nucl. Phys. B323, 37 (1989).

[2] J.P. Blaizot, A.H. Mueller, Nucl. Phys. B289, 847 (1987).

[3] X.-N. Wang and M. Gyulassy, Phys. Rev. D 44, 3501 (1991); Phys. Rev. D 45, 844 (1992).

[4] K. Geiger and B. Müller, Nucl. Phys. B369, 600(1992); K. Geiger, Phys. Rev. D 47, 133 (1993).

[5] I. Kawrakow, H.-J. Möhring, and J. Ranft, Nucl. Phys. A544, 471c (1992).

[6] J. Collins, D. E. Soper, and G. Sterman, Nucl. Phys. B261, 104 (1985).

[7] T. Sjöstrand and M. van Zijl, Phys. Rev. D 36, 2019 (1987); T. Sjöstrand, Comput. Phys. Commun. 39, 347 (1986); T. Sjöstrand and M. Bengtsson, ibid. 43, 367 (1987).

[8] T. B. Biró, E. van Doorn, B. Müller, T. H. Thoma, and X. N. Wang, Duke University preprint DUKE-TH-93-46, Phys. Rev. C in press.

[9] K. J. Eskola and M. Gyulassy, Phys. Rev. C 47, 2329 (1993).

[10] E. Eichten, I. Hinchliffe, K. Lane, and C. Quigg, Rev. Mod. Phys. 56, 579 (1984).

[11] G. Altarelli and G. Parisi, Nucl. Phys. B126, 298 (1977).

[12] A. Capella and J. Tran Thanh Van, Z. Phys. C 23, 165 (1984).

[13] P. l'Heureux, et al., Phys. Rev. D 32, 1681 (1985).

[14] G. Pancheri and Y. N. Srivastava, Phys. Lett. B 182, 199 (1986).

[15] L. Durand and H. Pi, Phys. Rev. Lett. 58, 303 (1987); Phys. Rev. D 38, 78 (1988).

[16] J. Dias de Deus and J. Kwiecinski, Phys. Lett. B 196, 537 (1987).

[17] T. K. Gaisser and F. Halzen, Phys. Rev. Lett. 54, 1754 (1985).

[18] X. N. Wang, Phys. Rev. D 43, 104 (1991).

[19] X.-N. Wang, Phys. Rev. D 46, R1900 (1992); D 47, 2754 (1993). 
[20] K. J. Eskola, LBL preprint LBL-32339,1992, Nucl. Phys. B in press.

[21] See e.g. R. D. Field, Applications of Perturbative QCD, Frontiers in Physics, Vol. 77 (Addison-Wesley, 1989).

[22] R. Odorico, Nucl. Phys. B172, 157 (1980).

[23] G. Marchesini and B. R. Webber, Nucl. Phys. B238, 1 (1984).

[24] A. Mueller, Nucl. Phys. A498, 41c (1988).

[25] D. W. Duke and J. F. Owens, Phys. Rev. D 30, 50 (1984).

[26] J. D. Bjorken, Phys. Rev. D 27, 140 (1983).

[27] E. Shuryak, Phys. Rev. Lett. 68, 3270 (1992).

[28] R. C. Hwa and K. Kajantie, Phys. Rev. Lett. 56, 696 (1986).

[29] J. Qiu and G. Sterman, Nucl. Phys. B353, 105 (1991).

[30] G. T. Bodwin, S. J. Brodsky and G. P. Lepage, Phys. Rev. D 39, 3287 (1989).

[31] P. Bordalo et al., Phys. Lett. B 193, 373 (1987); D. M. Alde et atl., Phys. Rev. Lett. 64, 2479 (1990).

[32] P. B. Straub et al., Phys. Rev. Lett. 68, 452 (1992).

[33] K. J. Eskola, J. Qiu and X.-N. Wang, LBL preprint, LBL-34163.

[34] T. Biró, B. Müller, and X.-N. Wang, Phys. Lett. B283, 171 (1992). 
FIG. 1. Illustration of a hard scattering and the associated branching trees. The values of $x_{1}$ and $x_{2}$ are the fractional energy carried by the in-coming partons. The number for each intermediate line is the life-time of that virtual partons $\Delta t=q_{0} / q^{2}(\mathrm{fm})$, and the numbers in the parentheses are the corresponding virtualities $q(\mathrm{GeV})$.

FIG. 2. The overlap region in space and time of two incoming partons each with spatial spread of $\Delta z_{1}$ and $\Delta z_{2}$, respectively. The interaction point is chosen randomly inside the shaded region. Solid lines show the trajectories of the parent nucleons.

FIG. 3. The total number of produced partons $N$ as a function of time $t$, with $t=0$ defined as when the two nuclei have complete overlap.

FIG. 4. The rapidity distribution $d N / d y$ of produced partons at different times $t$ (as indicated by the number for each line).

FIG. 5. $p_{T}$ distributions of produced partons at different times.

FIG. 6. Parton distribution along the $z$-axis at different times.

FIG. 7. Contour plot in $z-t$ plane of the parton density $\rho$ of Eq. 13, as indicated by the numbers. The wavy structure along the light-cone is only an artifact of the plotting program.

FIG. 8. Rapidity distributions of partons in the central slice of $|z|<0.5 \mathrm{fm}$ at different times.

FIG. 9. $p_{x}$ and $p_{z}$ distributions at different times for partons in the central slice $|z|<0.5$ fm. 
time for $A u+A u$ (solid)collisions, $A u+A u$ without radiations (dashed), and $p+p$ without radiation (dot-dashed).

FIG. 11. The final fractional number of produced quarks and anti-quarks as functions of rapidity for $A u+A u$ (solid)collisions, $A u+A u$ without radiations (dashed), and $p+p$ without radiation (dot-dashed).

FIG. 12. An illustration of the possible correlation between two initial branching trees leading to two hard scatterings. The two branching trees can result from parton splitting (fission) and recombination (fusion). The boxes represent hard processes with momentum scale $Q$. 


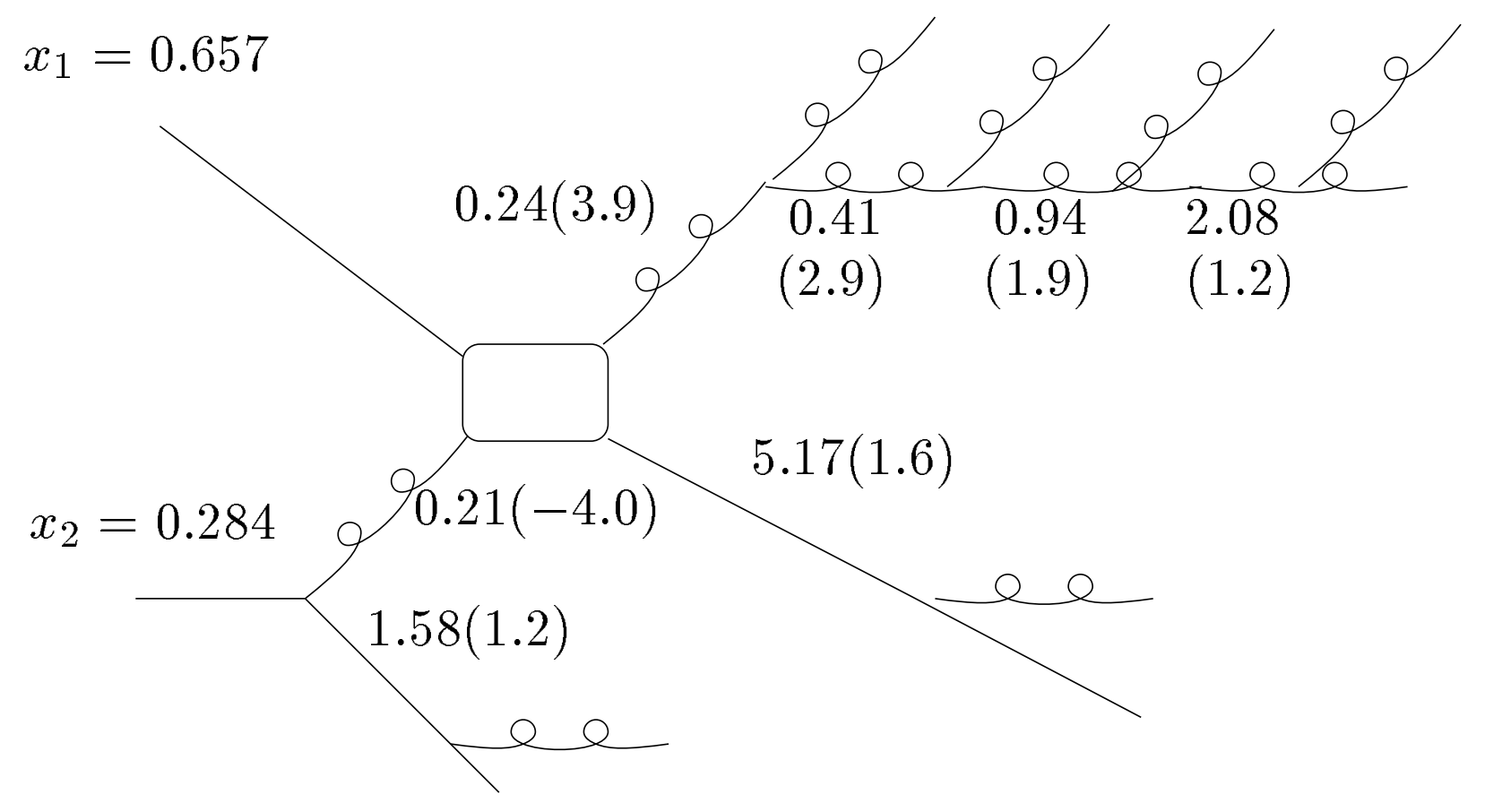

Fig. 1 


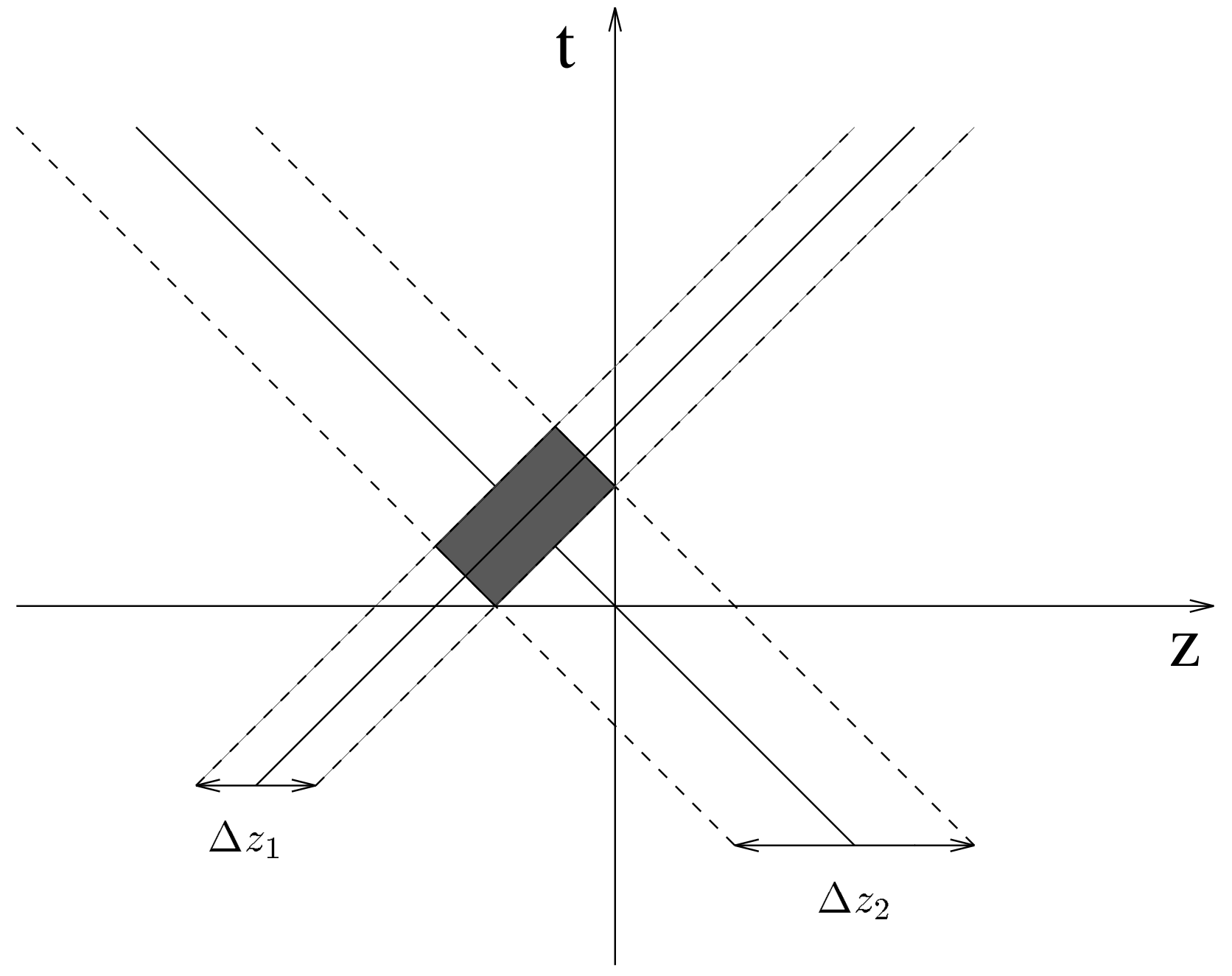

Fig. 2 


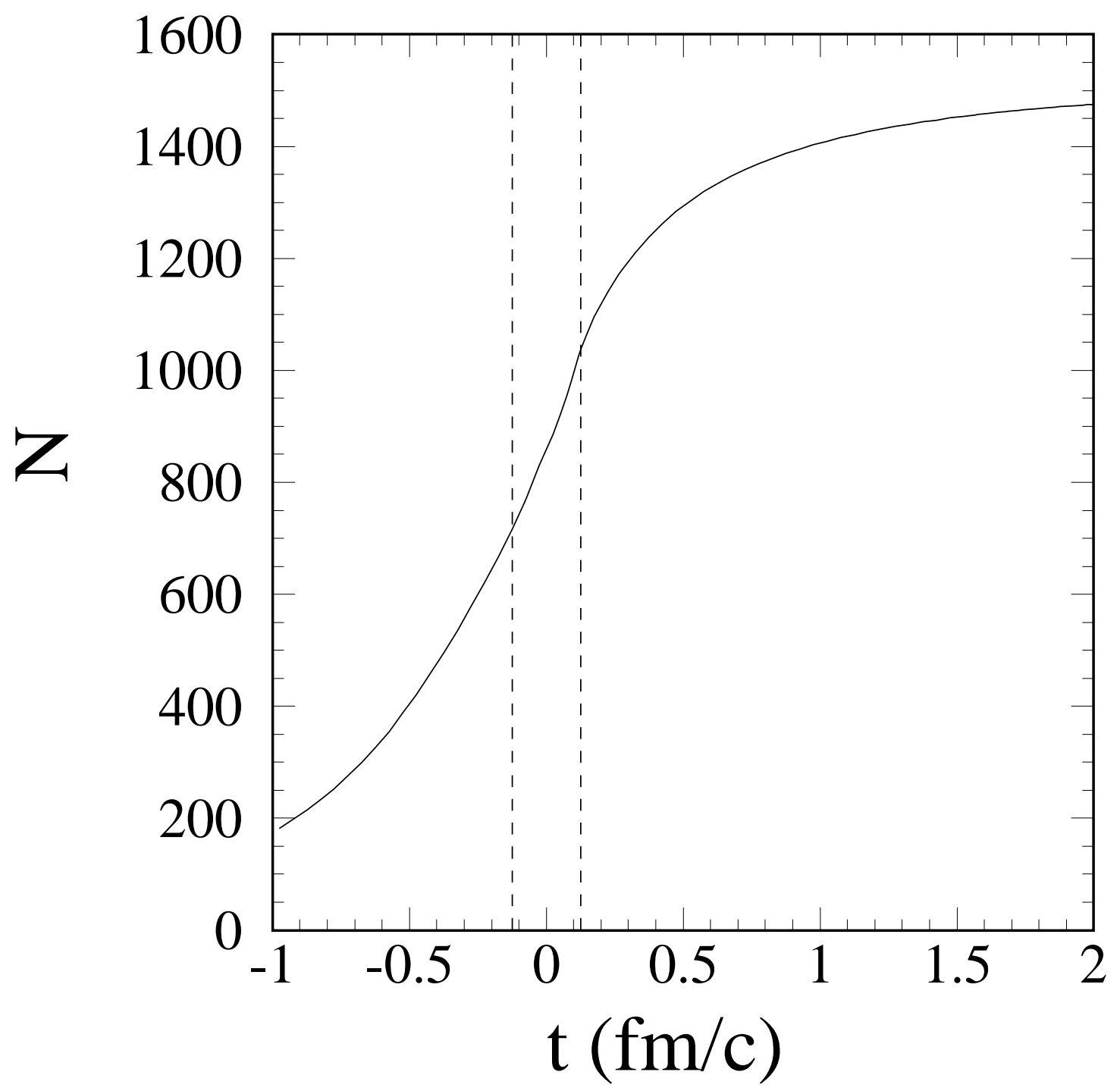

Fig. 3 


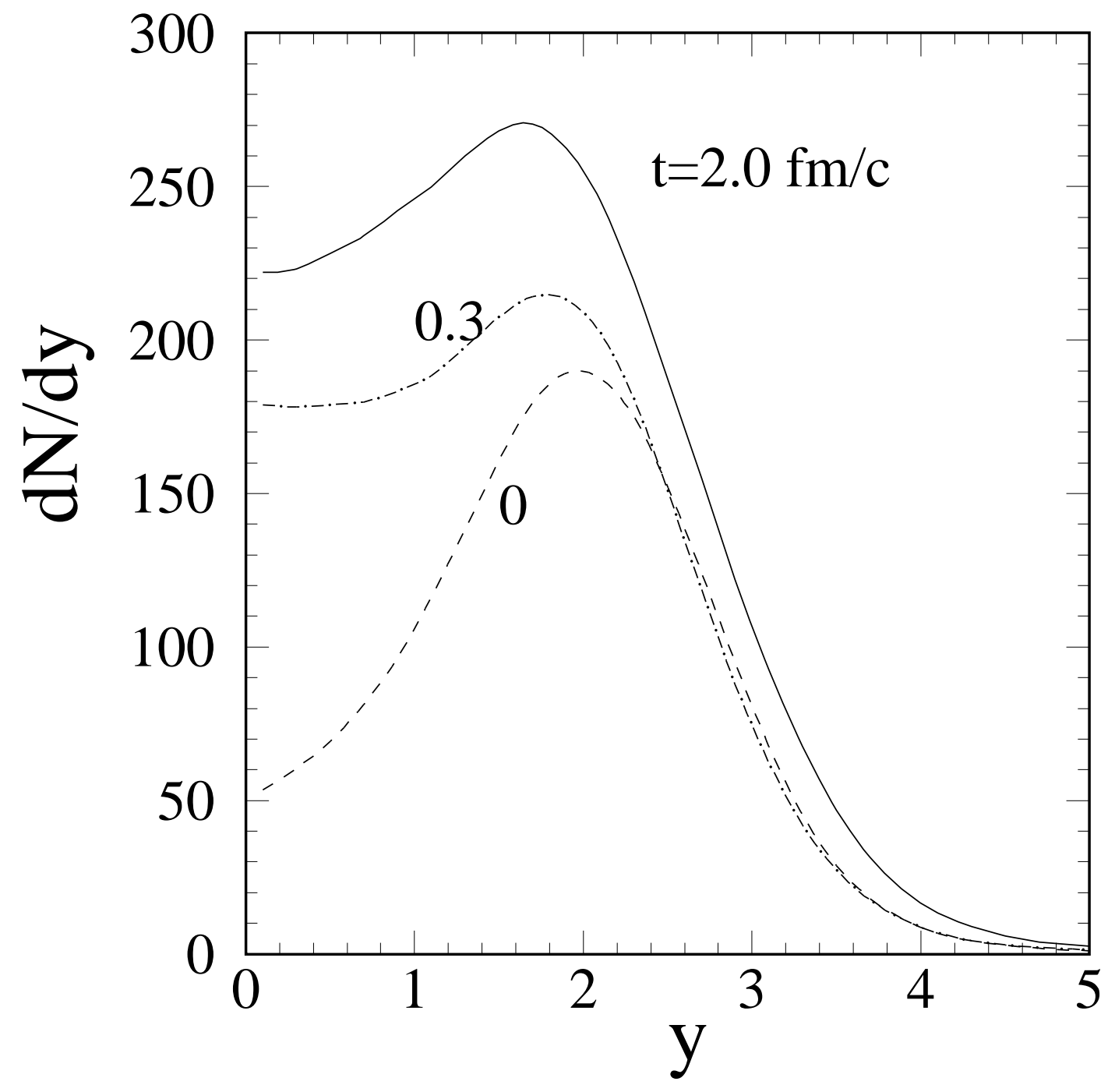

Fig. 4 


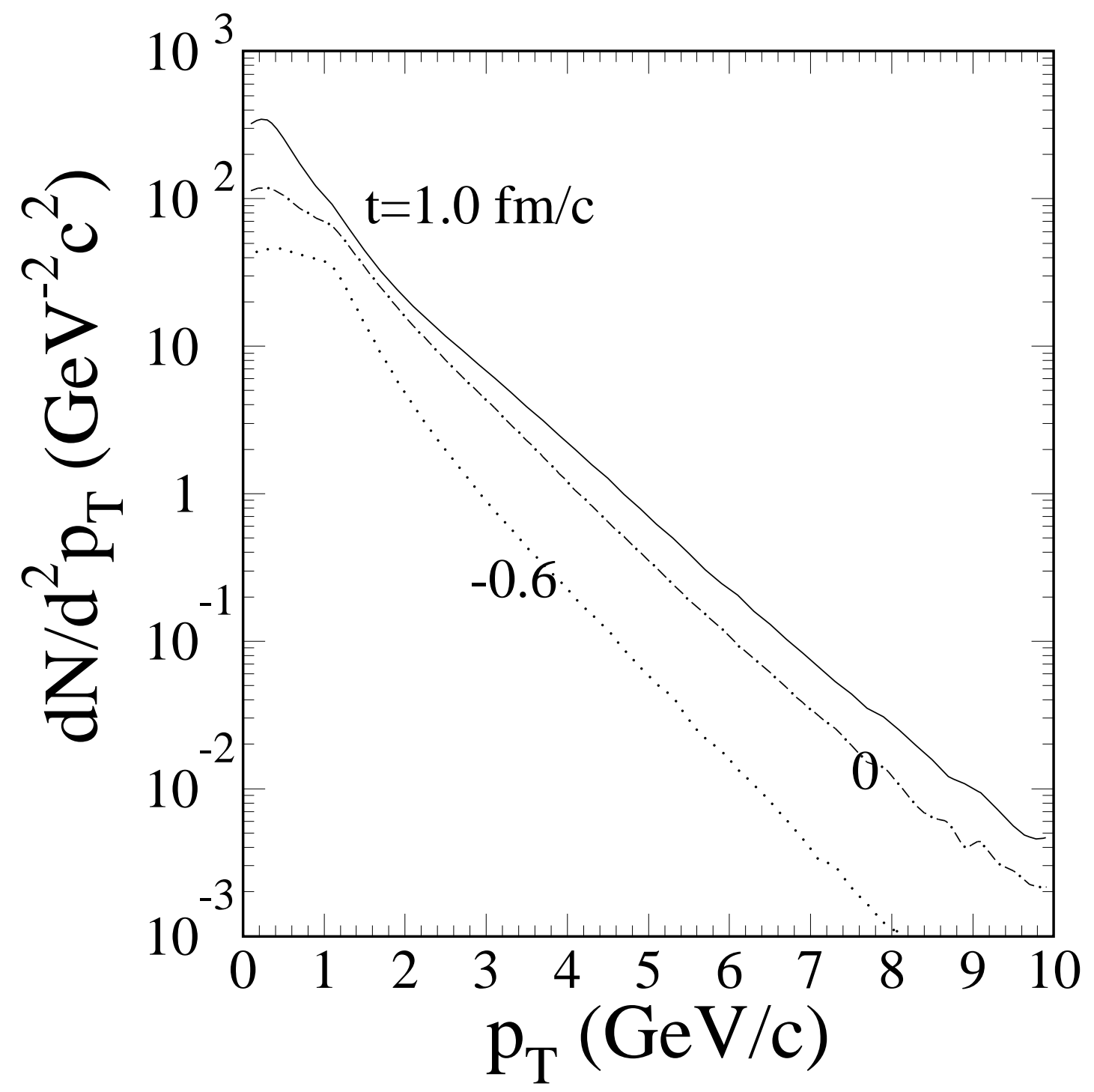

Fig. 5 


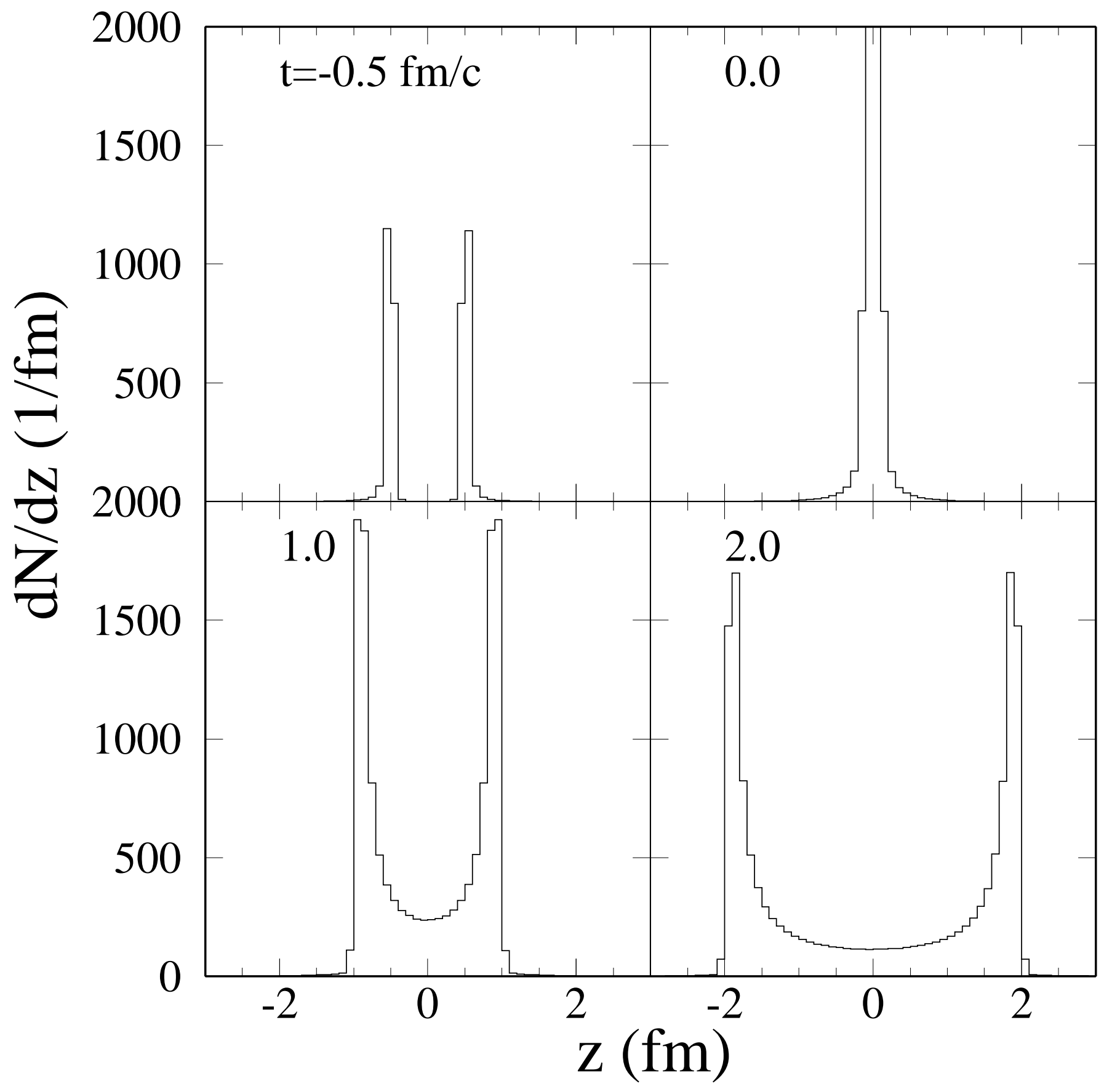

Fig. 6 


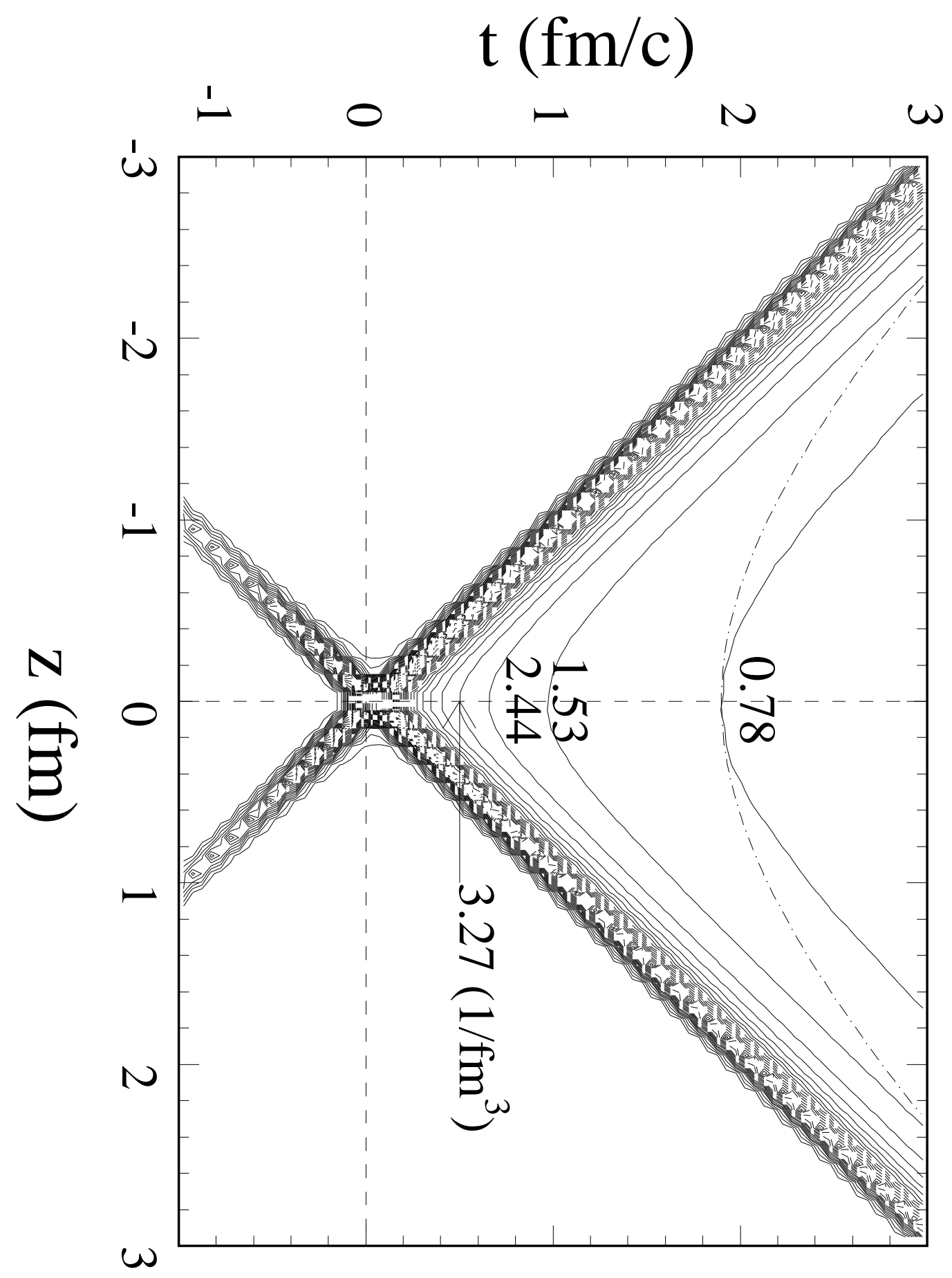

Fig. 7 


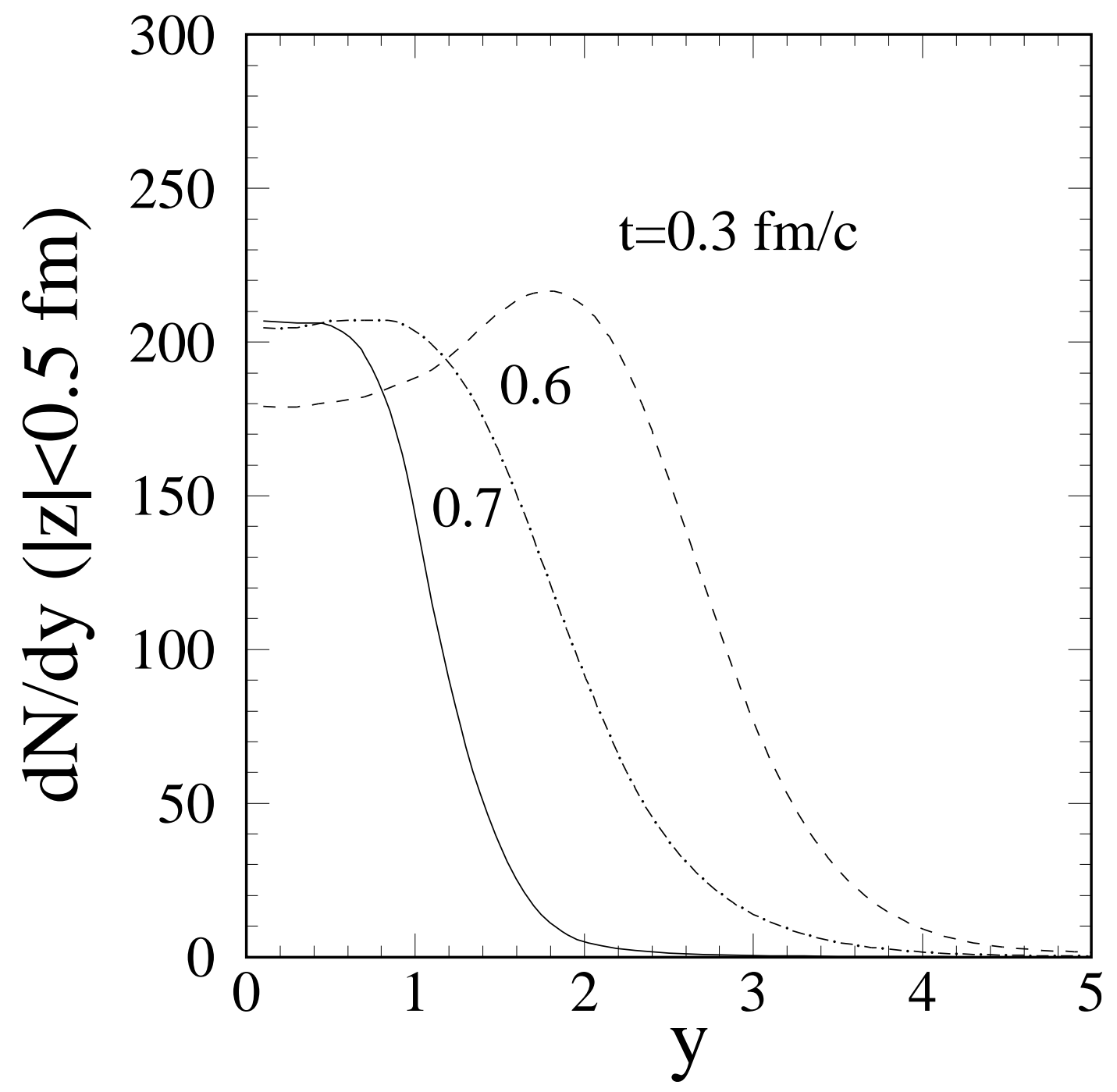

Fig. 8 


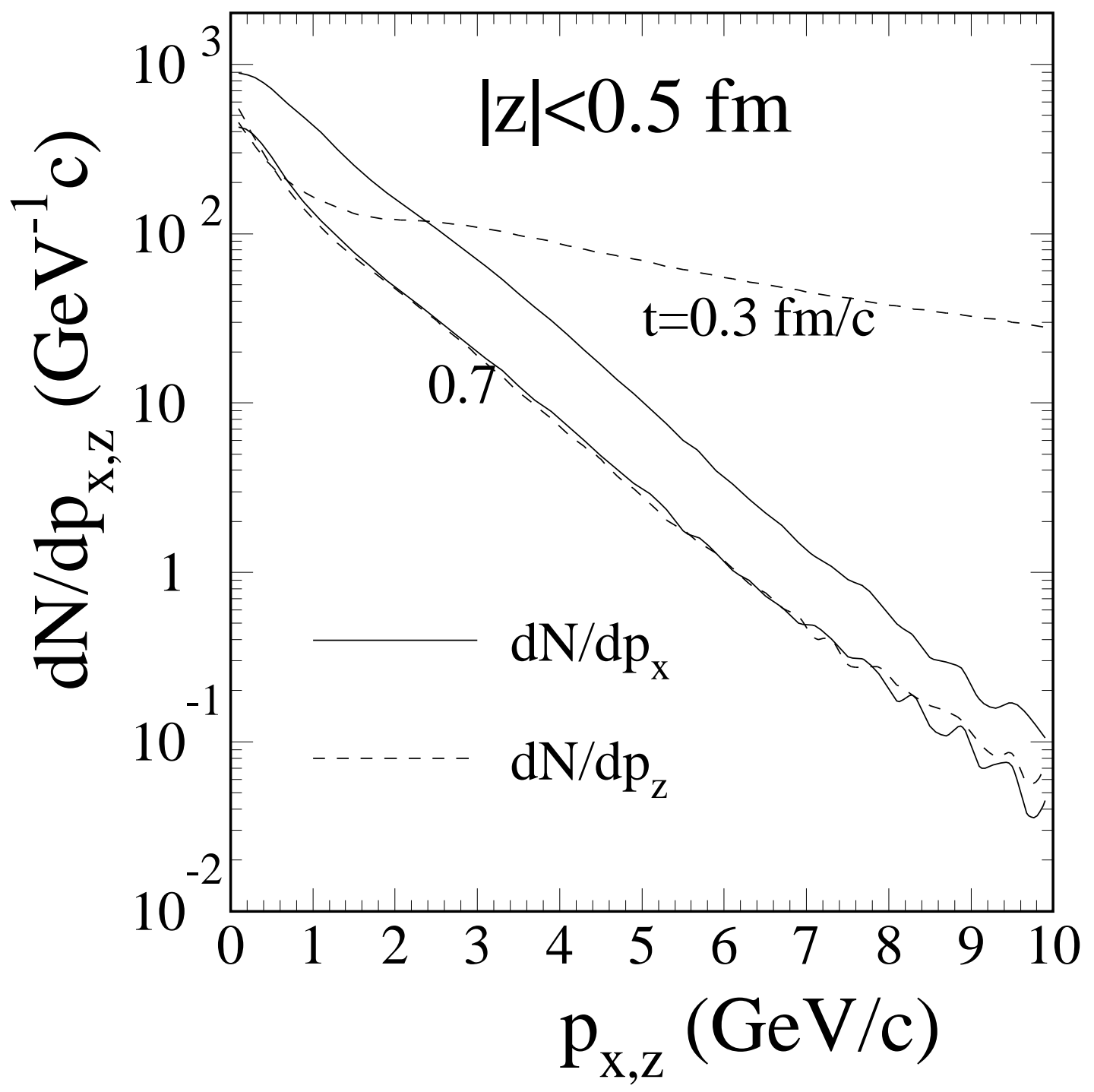

Fig. 9 


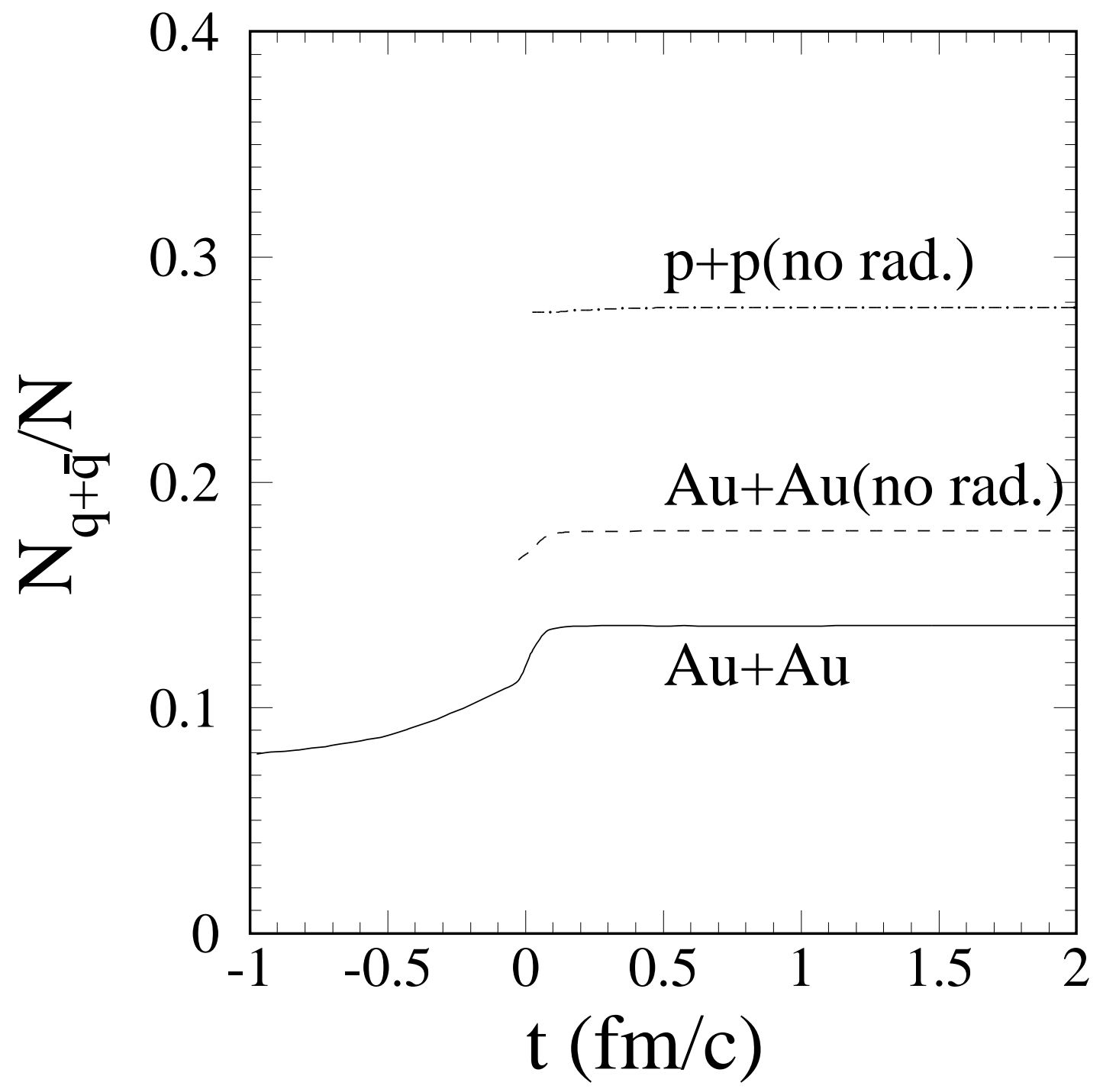

Fig. 10 


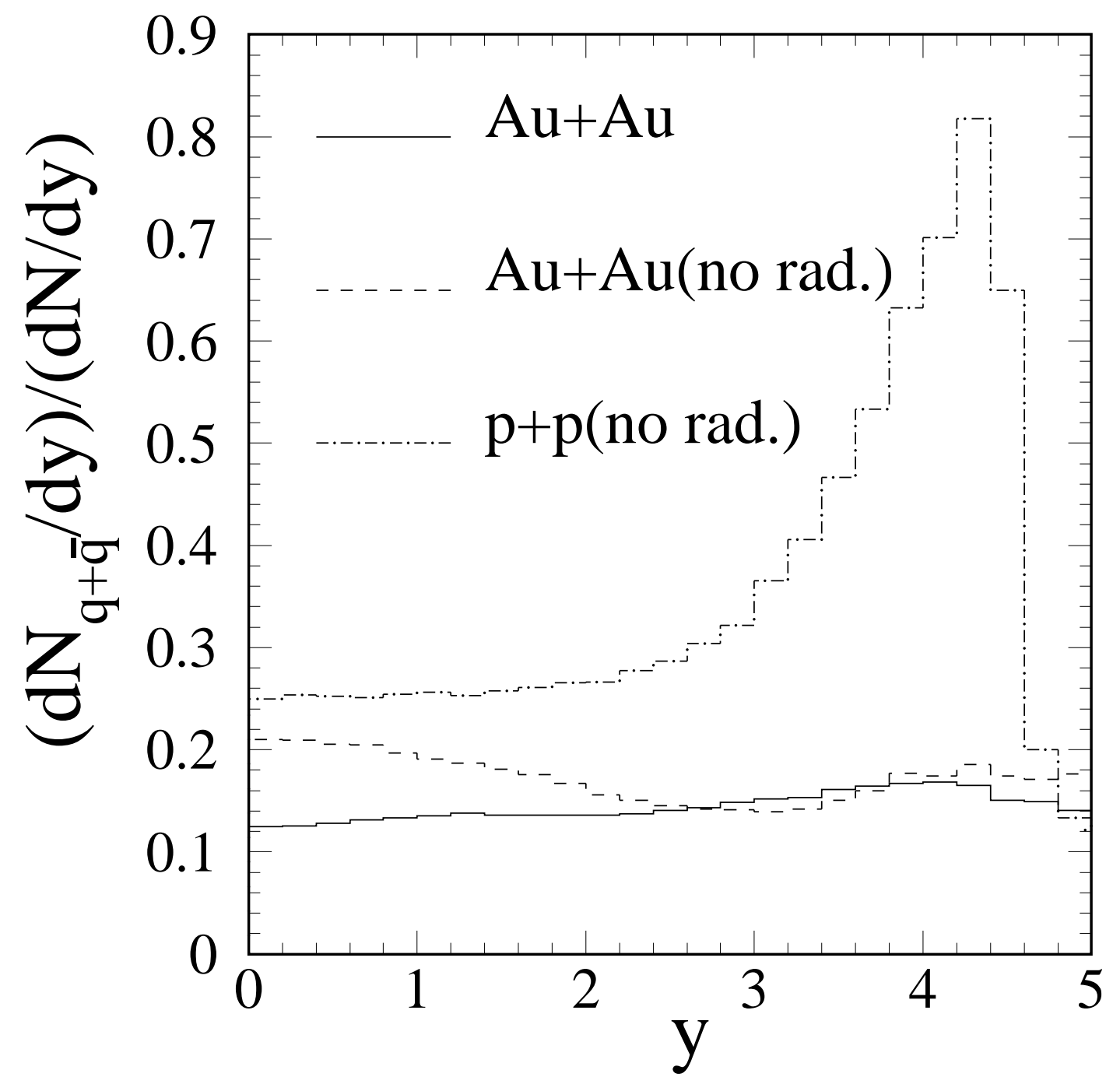

Fig. 11 


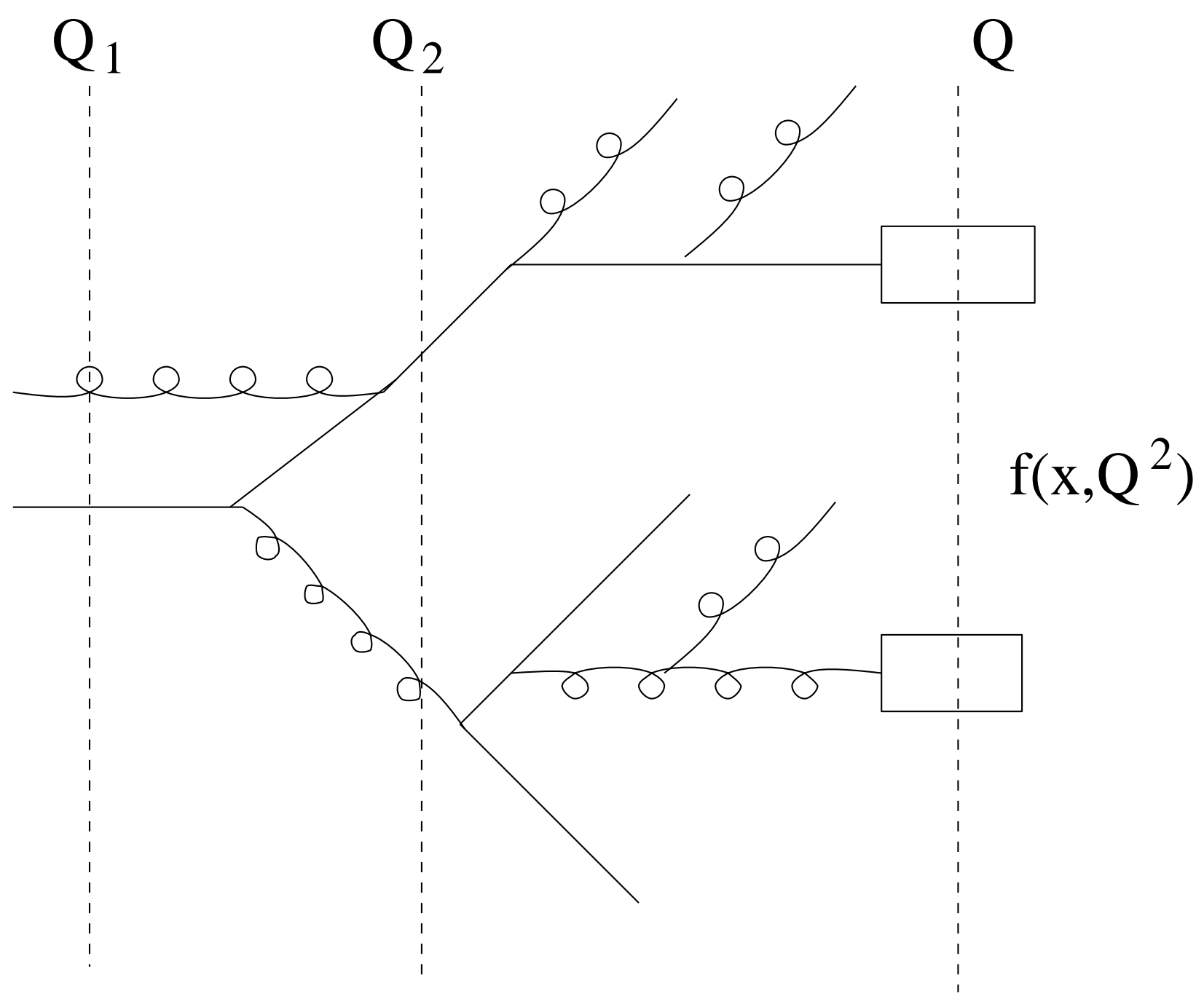

Fig. 12 